\title{
Costly Rewards and Punishments
}

\author{
Frances Xu Lee \\ Loyola University Chicago, flee3@luc.edu
}

Follow this and additional works at: https://ecommons.luc.edu/business_facpubs

Part of the Business Commons

\section{Recommended Citation}

Xu Lee, Frances. Costly Rewards and Punishments. The B.E. Journal of Theoretical Economics, 20, 1: , 2019. Retrieved from Loyola eCommons, School of Business: Faculty Publications and Other Works, http://dx.doi.org/10.1515/bejte-2018-0131

This Article is brought to you for free and open access by the Faculty Publications and Other Works by Department at Loyola eCommons. It has been accepted for inclusion in School of Business: Faculty Publications and Other Works by an authorized administrator of Loyola eCommons. For more information, please contact ecommons@luc.edu.

\section{(c) (1) $\Theta \Theta$}

This work is licensed under a Creative Commons Attribution-Noncommercial-No Derivative Works 3.0 License. (c) Walter de Gruyter GmbH, Berlin/Boston, 2019. 
Frances Z. Xu Lee'

\title{
Costly Rewards and Punishments
}

\author{
${ }^{1}$ Quinlan School of Business, Loyola University of Chicago, 16 E Pearson St, Chicago, IL 60611, USA, E-mail: \\ francesxu312@gmail.com
}

\begin{abstract}
:
To punish an agent, the principal often incurs costs. I study a principal's least costly reward and punishment scheme for an agent whose effort the principal cannot observe. I find the principal's cost is sometimes minimized by using both costly rewards and costly punishments because (1) the agent has an outside option, or (2) a principal without commitment ability repeatedly interacts with the agent. I also find that when an agent's effort is better at increasing the probability of a good outcome for the principal, the agent's payoff may decrease, because the principal replaces rewards with punishments.
\end{abstract}

Keywords: costly punishment, principal-agent, moral hazard, outside option, credibility

JEL classification: D23, L20, D86

DOI: 10.1515/bejte-2018-0131

\section{Introduction}

The choice between reward and punishment as incentive devices has attracted attention from the literature. Past work has largely focused on rewards and punishments in the form of monetary transfers such as subsidy (reward) versus taxation (punishment) or a bonus (reward) versus a fine (punishment). ${ }^{1}$ Non-monetary rewards or punishments have received relatively little attention. There are abundant examples of non-monetary rewards and punishments. Innovations can be rewarded with patents. A child can be rewarded with treats for good performance. A manager can be stripped of authority for mistakes. A teenager can be grounded for disobeying parents.

A principal's costs differ when using a monetary versus a non-monetary punishment. Monetary punishments benefit a principal by transferring money from the agent to the principal, whereas non-monetary punishments are costly to principals. For example, when a parent assigns chores to discipline a child, the parent incurs the cost of actively monitoring the child to ensure the completion of the chores. Similarly, when a community punishes criminals, the community must pay for expensive jails. ${ }^{2}$ The literature has long recognized that monetary punishment is often not feasible, so the principal-agent literature often assumes limited liability. However, costly punishment is often feasible for the principal.

I examine the trade-off between rewards and punishments in a model with agent moral hazard and limited liability. ${ }^{3}$ In my model, both rewards and punishments are costly to the principal. I endogenize the magnitude of both rewards and punishments in two separate frameworks: (1) where the principal has the power to commit to a pre-specified, outcome-contingent rewards and punishments scheme; and (2) where the principal cannot commit but interacts repeatedly with the agent over time.

Punishment is not simply a lack of a reward. ${ }^{4}$ In my model, a principal minimizes costs by imposing no reward and no punishment on the agent (the "no-reward-no-punishment" benchmark). Sometimes, this benchmark is clear. For example, a teacher minimizes costs by giving no praise or reprimand to students. Similarly, a community minimizes costs by not locking any citizens up or giving out any accolade. To move off the noreward-no punishment benchmark, a principal must incur costs. For example, a principal must use a costly reward to move an agent's payoff above this benchmark, or a costly punishment to move an agent's payoff below this benchmark. The larger the magnitude of the move, the larger the cost to the principal.

Significantly, an agent's expectation may set the principal's cost of punishment, because an agent's resistance to punishment is based on the agent's expectation. For example, if working conditions are expected to be bad, asking the agents to share an office is not a punishment and will not be met with agents' protest, but if the working conditions are expected to be good, not providing the agents free smoothies will be considered a punishment.

By modeling the reward-punishment trade-off where a principal can commit to a pre-specified, outcomecontingent rewards and punishments scheme for the agent, I show the impact of the agent's outside option and the benefit of the agent's effort. 
First, I show that an agent's outside option plays an important role in determining the principal's optimal scheme i. e., should a principal reward, punish, or both. The economics literature on contracting commonly considers an agent's outside option because an agent must accept a principal's incentive scheme for the scheme to be binding. For example, an immigrant accepts the reward and punishment scheme of a country by choosing to immigrate to it, and a student accepts the reward and punishment rules set forth in a syllabus by enrolling in a course. In the traditional principal-agent model with only monetary transfers, the outside option can be normalized to zero and does not play a role in the analysis. Here, I show the outside option, relative to the no-reward-no-punishment payoff of the agent, plays an important role.

Second, I show that the benefit of an agent's effort has a non-monotonic impact on the agent's expected payoff. When the benefit is low, the principal optimally chooses not to use any punishment because the chance of having to execute it is too high. This gives the agent a payoff above the outside option, because a principal only executes rewards. However, when the benefit of an agent's effort is higher, the principal may shift to using both reward and punishment, keeping the agent's payoff the same as the payoff from the outside option. That is, a higher benefit from the agent's effort can lead to worse payoff for the agent. When the benefit is even higher, the principal uses only punishment again, but the agent's better ability to avoid the punishable outcome increases the agent's payoff above the outside option.

These insights regarding the outside option and the effort's benefit speak to some questions that have been raised in Wittman (1984):

Why do ordinary economic markets tend to rely on positive incentives (the carrot), while government tend to rely on coercion (the stick)? ... Why is productive labor rewarded in capitalist societies while non-productive labor is punished in feudal societies?

Wittman (1984) attributes these phenomena to a lower administrative cost of punishment when the enforcer has coercive power. My paper suggests that agents' outside option and effort-outcome correlation may be the answer. People have worse outside options when facing a coercive government than facing an employer and the output of a low-skill labor activity is more correlated with the effort than that of a high-skill activity (Corollary 1 and Corollary 2).

In some applications, the party that metes out rewards and punishments does not have commitment ability and rewards and punishments can be sustained through repeated interaction. The literature of relational contracting has long studied the credibility of a bonus. Since punishment here is also costly to the principal, the credibility of a punishment is also an issue. For example, Donald Trump's campaign promise to sanction Saudi Arabia by banning oil imports from it was viewed as an empty threat because of the US's dependence of daily imports of more than 7 million barrels of crude oil from abroad. ${ }^{5}$ Therefore, my paper then turns to a framework of repeated interaction between a patient principal and an impatient agent, where the principal cannot commit but chooses reward and punishment levels in each period in a long term relationship with the agent. I find a limit on the joint intensity of the reward and the punishment. Very high reward or punishment is not credible because it is too costly to the principal. I find the reward and punishment are substitutes in terms of their credibility: a more intense reward makes a punishment less credible and vice versa. This credibility constraint, when binding, forces the principal to use both reward and punishment to motivate the agent's effort. This analysis is especially relevant in the context of parenting where promises of very lavishing rewards or threats of very harsh punishments on a child are not viewed as credible by a child.

Non-monetary punishment has appeared in several other contexts in the literature. In repeated interaction among oligopolies, collusion are sustained by punishing the deviating competitor with non-cooperative behavior (Green and Porter 1984). The payoff during the punishment phase is worse to all the firms than the payoff in the cooperative phase. In this sense, this non-monetary punishment is costly to all. In Fong and Li (2017), the principal can suspend or terminate the agent which destroys total surplus, so suspension or termination is like a punishment with endogenous costs to the principal. They show that the optimal relational contract involves a "probation phase", followed by a "reward phase" or a "punishment phase", which reflects a general lesson in dynamic contracting that reward and punishment should be backloaded in repeated interactions. However, I assume that the agent is impatient so the optimal relational contract has a stationary feature. Another endogenously costly way to punish the agent is to limit the agent's authority when the agent is better informed than the principal, as in Baker, Gibbons, and Murphy (1999) and Li, Matouschek, and Powell (2017). My punishment takes a simpler form of entailing direct current period costs for the principal. The worker can also punish the manager for reneging on payments through a non-monetary punishment: reducing effort, which is not directly costly to the worker (Li and Matouschek 2013).

This paper contributes to the law and economics literature that compares the reward and the punishment. The past literature has been largely focused on their impacts on the receivers without considering the cost of the enforcers. Two papers are exceptions to this. Wittman (1984) incorporates a "court administrative cost" for a social planner in executing either a reward or a punishment, that is not related to the magnitude of the 
incentives. He observes that if the threat of a punishment successfully implements the efficient outcome then no administrative cost is incurred. Dari-Mattiacci and De Geest (2010) show that, when constrained by the same capacity of a reward or a punishment, a punishment can motivate more effort than a reward across agents or over time. The capacity in their setup can be interpreted as a form of cost: no cost below the capacity but an infinite cost above it. De Geest and Dari-Mattiacci (2013) raise some disadvantage of punishment when comparing with reward. First, hidden information of the suitability of the agent for some tasks means it might be useless to try to punish the agent for bad performance in some tasks. Second, the principal sometimes only needs a small subset of the agents to reach a certain goal. These papers all view reward and punishment as mutually exclusive choices. Instead, I show that a scheme that involves both might be optimal: punishing the bad outcome to some intensity as well as rewarding the good outcome to some intensity. A parent rewards a child with treats (costly to the parent's pocket as well as the child's health which the parent internalizes to some extent) for a good performance and punishes a child with time-outs or corporal punishments for a bad performance. The US government promises conditional foreign aids while threatens economic sanctions that are also costly to the US economy. The issue of credibility was not considered in the law and economics literature because the social planner who metes out rewards or punishments was also implicitly assumed to have full commitment power.

In the Organizational Economics literature, reward and punishment are typically monetary transfers, which means reward is directly costly to the principal but punishment is directly beneficial to the principal. See Levin (2003). Lazear (1995) points out that rewards are better when it is not clear what the best performance is and the punishments are better when the it is not clear what the worse performance is. In a setting of multiple workers, Kamijo (2016) studies which scheme, rewarding the top performer or punishing the bottom performer, is better at motivating the workers taking into account the strategic interactions among the workers. In his paper, the intensity of the reward and punishment are fixed to be the same and their costs are not a consideration. Leshem and Tabbach (2015) study the design of reward and punishment in a "volunteer dilemma" where only one volunteer is needed. Their rewards and punishments are monetary transfers and thus only the reward is directly costly but punishment can be indirectly costly if it motivates too much volunteering.

If one interprets the principal as a parent and the agent as a child, my paper is related to studies on parenting strategies. ${ }^{6}$ Burton, Phipps, and Curtis (2002) document empirical evidences and argue that the parenting strategy (the amount of praise or scolding as a function of the child's behavior) should be modelled as being chosen according to the characteristics of the child.

The rest of the paper is organized as follows. Section 2 derives the least costly way to implement the effort in a full-commitment model with a sometimes binding outside option for the agent. It investigates how the intensity and the choice over the reward, punishment or both change with the outside option and the benefit of the effort. Section 3 studies a repeated interaction model without commitment to show that, in the absence of a binding outside option, mixing the reward and punishment can be generated by the credibility concern and the reward and punishment are substitutes in maintaining credibility. Section 4 discusses the issue of whether the principal wants to induce effort, altruistic principal and convex costs of rewarding and punishing the agent. Section 5 offers concluding remarks.

\section{Full Commitment Model with Agent Outside Option}

An outcome is either good or bad: $\left\{o_{1}, o_{2}\right\}$ with $o_{2}$ being the good outcome. ${ }^{7}$ An agent can exert a hidden effort to improve the probability of the good outcome. The effort $\operatorname{costs} c>0$ to the agent and increases the chance of a good outcome by $\theta>0$ (the benefit of effort). Without the effort, the chance of the good outcome is $p \in(0,1-\theta)$. The bad outcome's value to the principal is $v$, and the good outcome's value to the principal is $v+v$ with $v>0$. Here, I assume that $v$ is sufficiently high that the principal will choose to offer a scheme that motivates effort. ${ }^{8}$ All parameters are common knowledge.

A principal can control the agent's payoff, denoted by $x_{i}$ for being contingent on outcome $i \in\{1,2\}$, through a scheme. The principal's cost of implementing an agent payoff $x$ is denoted by function $k(x)$. It reaches its minimum at an interior level which I normalize to $x=0$. That is, we can think of the principal as directly choosing the amount of deviation from this benchmark level. A payoff above this level $\left(x_{i}>0\right)$ is a "reward" to the agent and a payoff below this level $\left(x_{i}<0\right)$ is a "punishment". The case of linear costs of rewarding and punishing the agent is the most interesting because it highlights the trade-off and it contrasts well with the traditional principalagent model. ${ }^{9}$ In the traditional principal-agent model where the principal uses direct monetary transfers, the cost of rewarding is directly the amount of monetary reward itself and thus linear. Linearity of the principal's cost technology amounts to the following assumption:

\section{Assumption 1.}


The principal's cost of giving agent payoff $x$ is:

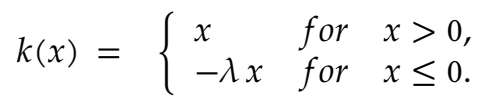

where $\lambda>0$.

Here the parameter $\lambda$ controls the relative costliness of the punishment versus the reward. If $\lambda>1$, per unit of change in the agent's payoff, the punishment is more expensive to the principal than the reward. If $\lambda<1$, it is the opposite. What is new here is that the principal's cost is not always decreasing in the agent payoff, which reflects costly punishment. I have maintained the limited liability assumption: the principal does not have a way to reduce the agent's payoff while benefiting itself directly, such as taking a monetary transfer from the agent.

The timing is the following: (1) The principal offers a scheme that maps the binary outcome to reward or punishment levels (intensities). (2) The agent chooses whether or not to accept the offer. The game ends if the offer is rejected. (3) The agent chooses whether or not to exert effort if the offer has been accepted. (4) Outcome realizes and punishment or reward is executed.

If the agent accepts the incentive scheme, his payoff is $x_{i}$ minus the effort cost if any. If the agent turns down the offer, he gets the outside option $t$ with $t \in(-\infty, \infty)$. I allow $t$ to be positive or negative, so the outside option can be better or worse than the no-effort and no-reward-no-punishment outcome for the agent. Let the principal's outside option be $\underline{w}$. The principal's payoff is the value derived from the outcome minus any reward/punishment costs. Assume $\underline{v}+p v>\underline{w}+t$ so the principal-agent relationship is worthwhile in the absence of any effort, reward or punishment. All players are risk neutral and maximize expected payoffs. I adopt Subgame Perfect Equilibrium.

I study the least costly scheme for the principal to induce the agent to accept the incentive scheme and exert effort, which I call the "optimal scheme" and I discuss when the principal will find it worthwhile to induce the agent effort in the discussion section Section 4.1.

The principal's optimal scheme is the solution to:

$$
\begin{aligned}
& \min _{x_{1}, x_{2}}\left\{\begin{array}{cc}
(1-p-\theta) x_{1}+(p+\theta) x_{2} & \text { if } x_{1} \geq 0, x_{2} \geq 0 \\
(1-p-\theta) \lambda\left(-x_{1}\right)+(p+\theta) x_{2} & \text { if } x_{1} \leq 0, x_{2} \geq 0 \\
(1-p-\theta) \lambda\left(-x_{1}\right)+(p+\theta) \lambda\left(-x_{2}\right) & \text { if } x_{1} \leq 0, x_{2} \leq 0
\end{array}\right. \\
& \text { s.t. } \quad(1-p-\theta) x_{1}+(p+\theta) x_{2}-c \geq t
\end{aligned}
$$

Standard in the principal-agent literature, there is an incentive compatibility constraint IC that says a good outcome should give the agent a payoff high enough above a bad outcome to induce effort. There is also an individual rationality constraint IR that says the agent's payoff of accepting the incentive scheme should be at least as high as the agent's outside option. In the objective function, the case of $x_{1} \geq 0$ and $x_{2} \leq 0$ (rewarding the bad outcome and punishing the good outcome) is omitted because it will clearly violate the IC constraint. Some terminology is in order. I will use the term "pure reward" for a scheme where a positive reward is given for the good outcome and no punishment is given for the bad. Similarly, a "pure punishment" is a scheme where a punishment is given for the bad outcome and no reward is given to the good outcome. A "mixed scheme" is one where a punishment is given for the bad outcome and a reward is given for the good. A "double reward" is one where a reward is given to both good and bad outcomes and a "double punishment" is one where a punishment is given to both good and bad outcomes. The following lemma simplifies the analysis to focus on the most relevant decisions: whether or not to punish the bad outcome and whether or not to reward the good outcome.

\section{Lemma 1.}

A scheme with $x_{1}<0$ and $x_{2}<0$ (double punishment) can never be the optimal scheme. If a scheme with $x_{1}>0$ and $x_{2}>0$ (double reward) is the optimal scheme, then IR must bind and also a scheme with $x_{1}^{\prime}=0$ and $x_{2}^{\prime}>0$ (pure reward) will also be the optimal scheme.

From the perspective of IC alone, it is never optimal to give costly punishment to the good outcome or to give costly reward to the bad outcome. However, doing the latter may be justified by the need to meet IR. The proof of Lemma 1 is in the Appendix.

I now simplify the principal's problem in minimizing the cost of implementing the effort by restricting to the domain of $x_{1} \leq 0$ and $x_{2} \geq 0$, keeping in mind that Lemma 1 implies that if a pure reward scheme is optimal with IR binding and IC lax, then a continuum of double reward schemes is also optimal. 
The principal's optimal scheme is the solution to:

$$
\begin{array}{lll}
\min _{x_{1}, x_{2}} & (1-p-\theta) \lambda\left(-x_{1}\right)+(p+\theta) x_{2} \\
\text { s.t. } & \theta\left(x_{2}-x_{1}\right) \geq c & \text { (IC) } \\
& (1-p-\theta) x_{1}+(p+\theta) x_{2}-c \geq t & \text { (IR) } \\
& x_{1} \leq 0 \text { and } x_{2} \geq 0
\end{array}
$$

Both punishment and reward contribute to meeting the IC constraint. The costly nature of the punishment makes punishment and reward substitutes in providing the incentive for the agent to exert effort. The ex ante expected marginal cost of punishment to the principal is $(1-p-\theta) \lambda$, while the expected marginal cost of reward to the principal is $(p+\theta)$. When $\theta$ is high, the chance of the punishment being executed is low while the chance of the reward being executed is high. Therefore, when $\theta$ is high it is more economical for the principal to use punishment to satisfy the agent's IC constraint and the opposite is true when $\theta$ is low, with the cutoff value for $\theta$ being $\frac{\lambda}{1+\lambda}-p$.

When $\theta<\frac{\lambda}{1+\lambda}-p$, the principal has a preference for using reward to fulfill IC. When the outside option is sufficiently good, the pure reward is set to meet IR, with IC being lax. When the outside option is worse, the pure reward is set to meet IC, with IR being lax.

On the other hand, when $1-p \geq \theta>\frac{\lambda}{1+\lambda}-p$, the principal has a preference for using punishment to fulfill IC. However, when the outside option $t$ is sufficiently attractive, the principal has to reply on a reward to satisfy agent's IR constraint. The optimal scheme is a pure reward to meet the binding IR and IC will be left lax. When the outside option is in an intermediate range, despite the principal's preference for using punishment to meet IC, she has to use some reward to meet IR. Both IR and IC are binding and the scheme is a mixed one. When the outside option is sufficiently unattractive, the principal has no fear of the agent quitting the relationship. She uses pure punishment to meet the binding IC with IR being lax. This is a simple linear programming problem that is best solved using graphs in the $\left(-x_{1}\right)-x_{2}$ space, which I omit. When IR is binding, punishment and reward form a complement to each other because a higher punishment calls for a higher reward to fulfill the agent's ex ante IR. Proposition 1 summarizes the optimal scheme of implementing the effort.

\section{Proposition 1.}

The optimal scheme, i. e. the solution to Problem (*), is the following.

1. $\theta<\frac{\lambda}{1+\lambda}-p$. The scheme is pure reward.

a. If $t>\frac{p}{\theta} c$, then $x_{1}=0$ and $x_{2}=\frac{t+c}{p+\theta}, I R$ is binding and IC is lax.

b. If $t<\frac{p}{\theta} c$, then $x_{1}=0$ and $x_{2}=\frac{c}{\theta}$, IR is lax and IC is binding.

2. $\theta>\frac{\lambda}{1+\lambda}-p$. The form of the scheme depends on $t$.

a. If $t>\frac{p}{\theta} c$, then $x_{1}=0$ and $x_{2}=\frac{t+c}{p+\theta}$ (pure reward). IR is binding and IC is lax.

b. If $t \in\left(-\frac{1-p}{\theta} c, \frac{p}{\theta} c\right]$, then $x_{1}=-\left(\frac{p}{\theta} c-t\right)$ and $x_{2}=t+\frac{1-p}{\theta} c$ (reward and punishment) . Both IR and IC are binding.

c. If $t \leq-\frac{1-p}{\theta} c, x_{1}=-\frac{c}{\theta}$ and $x_{2}=0$ (pure punishment). IR is lax and IC is binding.

When a pure reward is optimal, there are also schemes that reward both outcomes that are equally optimal. This is because IC is lax, one can move a little bit of reward to the bad outcome, keeping the expected total reward the same and keeping IC still satisfied. In all other cases, the above optimal schemes are the only optimal ones.

To see the impact of costly punishment, consider the special case where the costly punishment is not available or equivalently the cost of punishment is prohibitively high $(\lambda \rightarrow \infty)$. Then, only the subcase 1 in the proposition remains. By comparing subcase 2 to subcase 1 in the proposition, one can see that the availability of costly punishment strictly reduces the principal's and the agent's payoff when $t<\frac{p}{\theta} c$, and makes no difference otherwise.

\section{Comparative Statics with respect to $t$}

The agent's outside option makes an important role in the form of the optimal scheme, and in particular whether punishment is used and how much punishment is used.

\section{Corollary 1.}

The intensity of the punishment in the optimal scheme is decreasing in the agent's outside option. 


\section{Proof.}

Punishment is only deplored in subcase 2 of Proposition 1 . The intensity of punishment is continuous in $t$. When $t \leq-\frac{1-p}{\theta} c$, the punishment intensity is constant at $\frac{c}{\theta}$ as $t$ increases. In the range $t \in\left(-\frac{1-p}{\theta} c, \frac{p}{\theta} c\right]$, the intensity is $\frac{p}{\theta} c-t$, which is strictly decreasing in $t$. When $t<\frac{p}{\theta} c$, the intensity stays constant at 0 as $t$ increases.

Intuitively, better outside option means the principal has to load more of the incentive into the reward rather than the punishment for the agent to be willing to accept the scheme. As mentioned in the introduction, this provides an intuitive explanation for the intense use of the punishment by the government or the parents because the receivers' outside option of immigrating to a different country or breaking out of a family offers a very low payoff.

\section{Comparative statics with respect to $\theta$}

Now I turn my attention to the role of the effort's benefit $\theta$. While there are many parameter cases, there is a general pattern of how the form of the scheme and the agent payoff change with $\theta$, summarized below:

\section{Corollary 2.}

For any $t<p c /\left(\frac{\lambda}{1+\lambda}-p\right)$, there are two cutoffs $0<\theta_{1} \leq \theta_{2} \leq 1-p$ that divide the parameter space for $\theta$ into three (possibly empty depending on the other parameters) ranges $\left[0, \theta_{1}\right],\left[\theta_{1}, \theta_{2}\right]$ and $\left[\theta_{2}, 1-p\right]$. Within the interior of the first range, the optimal scheme is pure reward; the agent's expected payoff decreases in $\theta$ and is above the outside option. Within the interior of the second range, the optimal scheme is a mixed one; the agent's expected payoff is constant at the outside option. Within the interior of the third range, the optimal scheme is pure punishment; the agent's expected payoff is increasing in $\theta$ and is above the outside option.

The proof is in the Appendix. As the benefit of the effort increases ( $\theta$ increases), the least costly way of implementing the effort involves decreasing the intensity of incentives: the magnitude of reward and punishment (if any) are decreasing because a lower intensity is sufficient to motivate effort. This means that in the range of pure reward (unless the outside option is very high), an agent's payoff decreases in $\theta$ because the reward for the good outcome becomes smaller. This also implies that in the range of pure punishment, an agent's payoff increases in $\theta$ because the punishment for the bad outcome becomes smaller and also the punishment is less likely to be executed. When the outside option is very high, the optimal scheme is pure reward regardless of $\theta$.

Moreover, when $\theta$ reaches the critical value of $\frac{\lambda}{1+\lambda}-p$ from below (unless the outside option is very high), there is a discrete drop in the reward and a discrete increase in the punishment, reflecting that it becomes cost effective to replace reward with punishment to motivate effort. This change of instrument from reward to punishment reduces the agent's payoff discretely.

For example, one can interpret the principal as a school that adopts customized classroom policies that are contingent upon each student's performance. Higher ability students' effort is better at converting a bad outcome to a good outcome. For an outside option that is moderately negative, all three ranges in Corollary 2 is non-empty, so my model implies that the middle-ability students are pushed to a payoff the same as their outside options. Lower-ability students are placed under pure reward schemes which always give them payoffs above their outside options. Top ones are under pure punishment schemes but they are so capable that they can often avoid the punishment by avoiding the bad outcomes and they also enjoy a payoff above their outside options. The middle ones are schooled with both rewards and punishments but they are the least happy ones. ${ }^{10}$

This parameter of the benefit of effort is also related to how closely the outcome reflects the effort. When the outcome more closely reflects the effort, $p$ is smaller and $\theta$ is bigger. In fact, in the extreme case when outcome is certain given the effort choice or the effort is directly observable, i. e. not exerting effort is the bad outcome, exerting effort is the good outcome, $p=0$ and $\theta=1$. Note that lower $p$ and higher $\theta$ make the condition $\theta>\frac{\lambda}{1+\lambda}-p$ easier to satisfy and thus favors punishment. Agent's effort tend to be more observable with lower skill tasks than with higher skill tasks. For example, whether a student has exerted effort or not is not completely clear given a test outcome, but whether a child has cleaned up the room or not directly reflects the effort. Therefore, lower skill level tasks are optimally incentivized with more punishment. As mentioned in the introduction, this also provides a partial explanation to the question raised in Wittman (1984): "Why is productive labor rewarded in capitalist societies while non-productive labor is punished in feudal societies?". 


\section{Credible Punishment and Reward with No Commitment}

In the static model, I assumed that the principal can commit to the incentive scheme. However, after a bad outcome occurs, a principal will be reluctant to carry out a punishment because of its costs and after a good outcome occurs, a principal will also be reluctant to give a reward because of its costs. The principal is especially unlikely to be able to commit when the execution is not very public, such as a parent toward a child. To quote Gans (2010) on parenting:

...we can be reluctant to enact them (punishments), even after some behavioral offense has been committed. But once you show that reluctance, the threat of a punishment just isn't credible...A child can understand whether a threat is really credible or not and adjust their behavior accordingly.

With the parent-child relationship in mind, I assume for this section that the principal and the agent are locked in this relationship or equivalently both parties' outside options are negative infinity. ${ }^{11}$ The principal and the agent interact on date $n=1,2, \ldots$. At each date $n$, the following happens. (1) The principal announces an incentive scheme $\left(x_{1}, x_{2}\right)$ to the agent, following the notation in the static setup. (2) The agent chooses whether or not to exert the hidden effort. (3) The outcomes realizes. (4) Observing the outcome, the principal decides whether to honor or renege on the contingent reward or punishment. The same timing repeats again for the next date. Assume the principal has a discount factor $\delta \in(0,1)$ while the agent is impatient and has a discount factor of 0 . Assume $p \in(0,1-\theta)$, so the outcome is not a perfect signal of whether the agent has exerted effort, i. e. the monitoring of the agent is imperfect. However, the monitoring of the principal is perfect: deviation from the announced incentive scheme is publicly observable. All other assumptions follow the static model. The equilibrium concept, following Levin (2003), is Perfect Public Equilibrium (PPE).

The lack of patience on the agent's part implies that without loss of generality the optimal scheme for the principal is stationary. ${ }^{12}$ Stationarity means the principal proposes the same $x_{1}$ and $x_{2}$ in each period. Following the static setup, it is without loss of generality to restrict $x_{1} \leq 0$ and $x_{2} \geq 0$.

Even though the agent is impatient, he can condition his effort choice on the observed public history and in particular he can punish the principal for observably reneging on a reward or a punishment. The most severe form of punishing the principal for reneging is playing the static no-effort Nash Equilibrium in every period where the principal will not carry out any reward or punishment and the agent will not exert effort. The long term relationship between the principal and agent lends some credibility to the incentive scheme because of this consequence if the principal reneges. It might seem a little strange that the agent will "punish" the principal for not punishing the agent, but it is reasonable because one can interpret it as the punishment completely losing its credibility once it is not used as threatened. That is, the agent no longer believes that he will ever be punished for a bad outcome and so the agent will never exert effort and the best response of the principal is to never punish again. Indeed, the worst fear of a parent, when disciplining a child, is that if she does not carry out the punishment this time, the threat will never work in the future.

A scheme $\left(x_{1}, x_{2}\right)$ is credible only if the principal is willing to carry out $x_{1}$ after a bad outcome and $x_{2}$ after a good outcome. The payoff of executing the punishment or reward in the current period (and sustaining effort in all future periods as a result) should be weakly better than the payoff of not carrying out the punishment or reward (and losing effort for the rest of the relationship):

$$
\begin{aligned}
-\lambda\left(-x_{1}\right)+\frac{\delta}{1-\delta}\left(\underline{v}+(p+\theta) v-(p+\theta) x_{2}-(1-p-\theta) \lambda\left(-x_{1}\right)\right) & \geq \frac{\delta}{1-\delta}(\underline{v}+p v) \\
-x_{2}+\frac{\delta}{1-\delta}\left(\underline{v}+(p+\theta) v-(p+\theta) x_{2}-(1-p-\theta) \lambda\left(-x_{1}\right)\right) & \geq \frac{\delta}{1-\delta}(\underline{v}+p v)
\end{aligned}
$$

The first constraint above says that the principal prefers paying the cost of the punishment now (after the current period outcome is realized) and sustaining effort in the entire future to losing effort in the entire future. The second constraint above says that the principal prefers paying the cost of the reward now and sustaining effort in the entire future to losing effort in the entire future. The two constraints are related. More intense future punishment makes the future of sustaining effort less attractive to the principal and puts a tighter credibility constraint on the intensity of the current period reward and vice versa. These two constraints can be combined into one credibility constraint:

$$
\max \left\{\lambda(1+\alpha)\left(-x_{1}\right)+\beta x_{2}, \lambda \alpha\left(-x_{1}\right)+(1+\beta) x_{2}\right\} \leq \gamma
$$

where $\alpha=\frac{\delta}{1-\delta}(1-p-\theta), \beta=\frac{\delta}{1-\delta}(p+\theta)$ and $\gamma=\frac{\delta}{1-\delta} \theta v$. This credibility constraint shows that the amounts of reward and punishment are substitutes in maintaining credibility. A higher amount of costly reward reduces the future value of sustaining effort (with the same amount of reward) for the principal, which in turn makes it harder to sustain a higher amount of punishment. 
The principal's optimal stationary scheme is the solution to:

$$
\begin{array}{ll}
\min _{x_{1}, x_{2}} & (1-p-\theta) \lambda\left(-x_{1}\right)+(p+\theta) x_{2} \\
\text { s.t. } & \theta\left(x_{2}-x_{1}\right) \geq c \\
& \max \left\{\lambda(1+\alpha)\left(-x_{1}\right)+\beta x_{2}, \lambda \alpha\left(-x_{1}\right)+(1+\beta) x_{2}\right\} \leq \gamma \\
& x_{1} \leq 0 \text { and } x_{2} \geq 0
\end{array}
$$

The only difference from Problem $\left(^{*}\right)$ in Section 2 is that the IR constraint is replaced by credibility constraints CC. Note that IC has to be binding at the optimal solution because otherwise $x_{1}=x_{2}=0$ is credible and cost minimizing. According to Proposition 1 in the static setup, IC alone implies that the optimal scheme that implements effort takes the following form:

1. When $\theta<\frac{\lambda}{1+\lambda}-p$, the optimal static scheme to implement effort is a pure reward where $x_{1}=0$ and $x_{2}=\frac{c}{\theta}$.

2. When $\theta>\frac{\lambda}{1+\lambda}-p$, the optimal static scheme to implement effort is a pure punishment where $x_{1}=-\frac{c}{\theta}$ and $x_{2}=0$.

Consider the first case $\left(\theta<\frac{\lambda}{1+\lambda}-p\right)$, illustrated by Figure 1 in the $\left(-x_{1}\right)-x_{2}$ space. The horizontal axis is the intensity of the punishment and the vertical axis is the intensity of the reward. The iso-cost line is steeper than IC, indicating that the figure is depicting the case where it is cheaper to use reward than punishment in expectation. A scheme satisfies the IC constraint if it is above the IC line in the figure and it satisfies the CC constraint if it is below the CC kinked line in the figure. Therefore, a feasible scheme is one in between the IC and CC lines in the figure. When IC does not intersect the kinked CC and is entirely below it, CC is not binding, one can load all the incentive onto a reward and still satisfy CC. That is, when $\frac{c}{\theta}<\frac{\gamma}{1+\beta}$, the solution is $x_{1}=0$ and $x_{2}=\frac{c}{\theta}$, as shown in Figure 1(a). When the IC line intersects CC, CC is binding and the solution is a mix of reward and punishment with $x_{1}<0$ and $x_{2}>0$, shown in Figure 1(b). Basically, the principal cannot load all the incentive into the reward because that high intensity of reward is not credible, so the principal has to use some punishment. When IC is too high, there is no feasible area in the $\left(-x_{1}\right)-x_{2}$ space that satisfies both IC and CC simultaneously, so no effort can be sustained. The other possible case $\left(\theta>\frac{\lambda}{1+\lambda}-p\right)$, where it is cheaper to use punishment than reward in expectation, is analogous. Therefore,

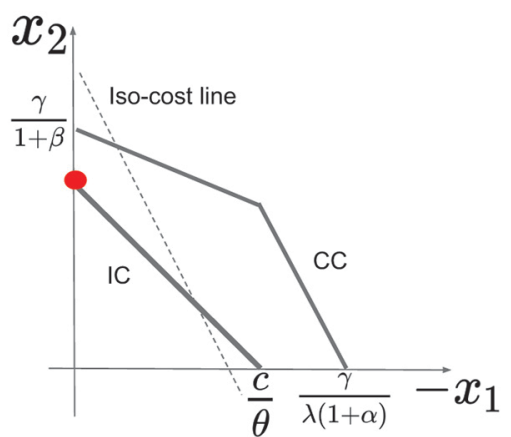

(a)

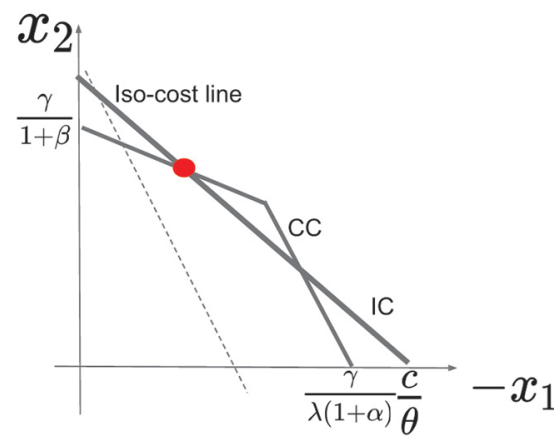

(b)

Figure 1: The optimal stationary scheme when reward is cheaper than punishment $\left(\theta<\frac{\lambda}{1+\lambda}-p\right)$.

Proposition 2.

In an infinitely repeated setup with no principal commitment ability, the optimal scheme is the following:

1. When $\theta<\frac{\lambda}{1+\lambda}-p$,

(a) When $\frac{c}{\theta} \leq \frac{\delta \theta v}{1-\delta(1-p-\theta)}$, the optimal stationary scheme to implement effort is $x_{1}=0$ and $x_{2}=\frac{c}{\theta}$ (pure reward).

(b) When $\frac{\delta \theta v}{1-\delta(1-p-\theta)}<\frac{c}{\theta} \leq \frac{1+\lambda}{\lambda} \delta \theta v$, the optimal stationary scheme to implement effort is $x_{1}=-\frac{(1-\delta(1-p-\theta)) \frac{c}{\theta}-\delta \theta v}{1-(1+\lambda) \delta(1-p-\theta)}$ and $x_{2}=\frac{\delta \theta v-\lambda \delta(1-p-\theta) \frac{c}{\theta}}{1-(1+\lambda) \delta(1-p-\theta)}$. (reward and punishment).

2. When $\theta>\frac{\lambda}{1+\lambda}-p$,

(a) When $\frac{c}{\theta} \leq \frac{\delta \theta v}{\lambda(1-\delta(p+\theta))}$, the optimal stationary scheme to implement effort is $x_{1}=-\frac{c}{\theta}$ and $x_{2}=0$ (pure punishment).

(b) When $\frac{\delta \theta v}{\lambda(1-\delta(p+\theta))}<\frac{c}{\theta} \leq \frac{1+\lambda}{\lambda} \delta \theta v$, the optimal stationary scheme to implement effort is $x_{1}=-\frac{\delta \theta v-\delta(p+\theta) \frac{c}{\theta}}{\lambda-\delta(1+\lambda)(p+\theta)}$ and $x_{2}=\frac{\lambda(1-\delta)(p+\theta) \frac{c}{\theta}-\delta \theta v}{\lambda-\delta(1+\lambda)(p+\theta)}$ (reward and punishment). 
When $\frac{c}{\theta}>\frac{1+\lambda}{\lambda} \delta \theta v$, effort cannot be implemented.

The proof is in the Appendix.

Here the pure reward and pure punishment schemes are optimal when the credibility constraint CC does not bind in 1 (a) and 1(b). This is when $\gamma=\frac{\delta}{1-\delta} \theta v$ (the present value of the effort) is relatively large compared to $\frac{c}{\theta}$. When $\gamma$ is smaller, the need to keep the reward and punishment credible sometimes generates the mixture of reward and punishment in the incentive scheme as shown in cases 2(a) and 2(b). When $\gamma$ is even smaller, there is no hope to sustain effort with any credible scheme.

\section{Comparative statics with respect to $\theta$}

An increase in $\theta$ in the repeated setting can change the amount of reward or punishment in a way similar to the static model. First, for a higher benefit of effort, a smaller amount of reward or punishment is needed to motivate, which is reflected in the expression $\frac{c}{\theta}$ that is decreasing in $\theta$. Second, similar to the static model, a higher benefit of effort may cause the principal to suddenly replace some reward with some punishment, as $\theta$ crosses the critical value $\frac{\lambda}{1+\lambda}-p$. This causes a discrete drop in the agent's payoff.

However, the credibility constraint in the repeated setup has a new implication on how $\theta$ will affect the intensities of reward and punishment and the agent's payoff. A higher $\theta$ relaxes the credibility constraint CC. This is because a more beneficial effort makes future efforts more valuable to the principal (reflected in a higher $\gamma$ ) and reduces future cost of punishments (reflected in a lower $\alpha$ ), both giving the principal more incentive not to renege on the reward or punishment in the current period. Even though a more beneficial effort also increases future cost of rewards (reflected in a higher $\beta$ ), this effect is dominated by the other two.

As a result, the comparative statics with respect to $\theta$ is more complicated in the repeated setting than in the static setting. In the static setting, given a sufficient low and thus irrelevant outside option, the form of the optimal scheme only changes once as $\theta$ increases: it switches from pure reward to pure punishment when $\theta$ crosses $\frac{\lambda}{1+\lambda}-p$ from below. Now, with the added credibility constraint, within the range $\theta<\frac{\lambda}{1+\lambda}-p$, the form of the optimal scheme may change from mixed to pure reward as $\theta$ increases. This is because for a low $\theta, C C$ is binding and forces the optimal scheme to load incentives into both reward and punishment. Similarly, within the range $\theta>\frac{\lambda}{1+\lambda}-p$, the form of the optimal scheme may change from mixed to pure punishment as $\theta$ increases. The richest possible variation in the form of the optimal scheme with respect to $\theta$ is the following:

In repeated interaction, when the benefit of the effort increases, the optimal scheme may go through four stages: mixed, then pure reward, then mixed again, and then pure punishment. For example, consider this numerical example: $c=1, \lambda=0.25, p=0, \delta=0.5, v=32$. Then for $\theta<0.11$, there is no scheme that can implement effort. For $0.11<\theta<0.19$, the optimal scheme is mixed. For $0.19<\theta<0.20$, the optimal scheme is pure reward. For $0.20<\theta<0.23$, the optimal scheme is mixed. For $\theta>0.23$, the optimal scheme is pure punishment. (All cutoffs are rounded to two digits after the decimal point.) The intuition is the following. (1) When $\theta$ is extremely small, there is no hope to motivate effort. (2) When $\theta$ is in the next higher range, reward is still cheaper in expectation, but the principal needs a high powered incentive that is only credible if it is loaded into both reward and punishment. (3) When $\theta$ is a little higher, the principal can load all the incentive into a reward. (4) When $\theta$ is even higher, punishment becomes cheaper in expectation, but the incentive needed is still big enough that it has to be loaded into both reward and punishment to be credible. (5) When $\theta$ is even higher, only a small incentive is needed and it can be loaded entirely into a punishment.

\section{Discussion}

\subsection{Principal's Incentive to Motivate Effort}

In the full-commitment setup of Section 2, to motivate effort a principal will use the scheme in Proposition 1, which I will refer to here as the effort scheme. To forgo the effort, the optimal scheme for the principal depends on the outside option. When the outside option of the agent is good $(t>0)$, a least costly way to keep the agent in the relationship without effort is to reward the agent $t$ regardless of the outcome. When the outside option of the agent is bad $(t<0)$, the principal cannot take advantage of the bad outside option because of the limited liability: the principal cannot charge the agent to accept the scheme, so the least costly way is to pay the agent 0 regardless of outcomes. Put in another way: there is a basic cost of keeping the agent in the relationship if $t>0$ at the level $t$. Otherwise, there is no cost in keeping the agent in the relationship. I will refer to the scheme that achieves this as the no-effort scheme. 
The principal compares the value of the effort $\theta v$ with the cost of motivating the effort, i. e. the cost under the effort scheme minus that under the no-effort scheme. This decision is not always efficient. Efficiency requires the effort to be exerted whenever $\theta v>c$. That is, the value of the effort to the principal exceeds the cost of effort to the agent. I say that there is too little incentive to motivate effort if the cost of motivating the effort for the principal is higher than $c$ and I say that there is too much incentive to motivate effort if the cost of motivating the effort is lower than $c$.

Without punishment as a tool, it is well known that there is sometimes too little incentive to motivate effort when there is limited liability. In my particular setup, the cutoff for the outside option is $\frac{p}{\theta} c$. For $t \geq \frac{p}{\theta} c$, the effort decision is always efficient because the limited liability is not binding. The principal keeps the agent at the outside option and thus internalizes the entire benefit of effort. For $t<\frac{p}{\theta} c$, the principal does not internalize the entire benefit of effort because the principal cannot charge the agent, so there is too little incentive to motivate effort. A similar intuition holds here, when reward is the only instrument used to motivate effort, the cutoff $\frac{p}{\theta} c$ determines whether there is too little or just the efficient amount of incentive to motivate effort.

However, when $\lambda$ is sufficiently low and outside option $t$ is not too good, the tool of punishment is deplored and it reduces the principal's cost of motivating effort. When this cost reduction is significant enough (i. e. when $\lambda$ is below some outside-option-dependent cutoff), there is too much incentive to motivate effort. Think of the extreme case where punishment is completely free: $\lambda=0$ and the outside option $t \leq-(1-p) c / \theta$. In this case, the principal will motivate effort with pure punishment regardless of whether effort is efficient or not. What is more interesting is that (1) even when the punishment is more costly than the reward in moving the agent's payoff, i. e. $\lambda>1$, the incentive to motivate effort may still be too high. This is because the expected per unit cost of using punishment $(1-p-\theta) \lambda$ might be sufficiently lower than the expected per unit cost of using reward $(p+\theta) ;(2)$ the agent's outside option being bad $(t<0)$ is a necessary condition for the incentive to motivate effort to be too high.

\section{Proposition 3.}

In the full-commitment setup,

1. When $t \geq \frac{p}{\theta}$ c, the effort is always efficient.

2. When $0<t<\frac{p}{\theta}$ c, there is too little incentive to motivate effort.

3. When $t<0$, there is too little incentive to motivate effort if $\lambda>\hat{\lambda}(t)$ and there is too much incentive to motivate effort if $\lambda<\hat{\lambda}(t)$.

\section{Proof.}

When $t>\frac{p}{\theta} c$, by 1(a) and 2(a) in Proposition 1, the optimal scheme is to use pure reward $x_{2}=\frac{t+c}{p+\theta}$, so the expected cost under the effort scheme is $c+t$. Since $t>0$, the expected cost of the no-effort scheme is $t$. Therefore, the cost of motivating effort is $c$. Therefore, the decision is always efficient.

When $0<t<\frac{p}{\theta} c$, there are two cases. Case 1. reward is cheaper in expectation: $\lambda>\frac{p+\theta}{1-p-\theta}$. In this case, pure reward is used with $x_{2}=\frac{c}{\theta}$, so the expected cost under the effort scheme is $(p+\theta) \frac{c}{\theta}=c+\frac{p}{\theta} c>c+t$. Since $t>0$, the expected cost of the no-effort scheme is $t$. Therefore, the cost of motivating effort is strictly greater than $c$. Therefore, there is too little incentive to motivate effort. Case 2. reward is more expensive in expectation: $\lambda<\frac{p+\theta}{1-p-\theta}$. In this case, reward and punishment are both used. The expected cost under the effort scheme, denoted by $K_{e}$, is strictly higher than the cost of the reward alone:

$$
K_{e}>(p+\theta)\left(t+\frac{1-p}{\theta} c\right)>c+t
$$

The second inequality is due to $t<\frac{p}{\theta} c$. Since $t>0$, the expected cost of the no-effort scheme is $t$. Therefore, the cost of motivating effort is strictly greater than $c$.

When $t<0$, the expected cost of the no-effort scheme is 0 , and there are two cases regarding the cost of the effort scheme. Case 1. reward is cheaper in expectation: $\lambda>\frac{p+\theta}{1-p-\theta}$. In this case, pure reward is used with $x_{2}=\frac{c}{\theta}$, so the expected cost under the effort scheme is $(p+\theta) \frac{c}{\theta}=c+\frac{p}{\theta} c$. Therefore, the cost of motivating effort is $c+\frac{p}{\theta} c>c$, so there is too little incentive to motivate effort. Case 2. reward is more expensive in expectation: $\lambda<\frac{p+\theta}{1-p-\theta}$. For $t>-\left(\frac{1-p}{\theta}-1\right) \frac{p}{p+\theta} c>-\frac{1-p}{\theta} c, 2(b)$ in Proposition 1 applies. Then for any $\lambda \geq 0, K_{e}>(p+\theta)(t+$ $\left.\frac{1-p}{\theta} c\right)>c$, so there is too little incentive to motivate effort. Let $K_{e}(\lambda)$ denote the cost under the effort scheme given different $\lambda$. For $t<-\left(\frac{1-p}{\theta}-1\right) \frac{p}{p+\theta} c, K_{e}(0)<c$. This is because either $t>-\frac{1-p}{\theta} c(2(b)$ in Proposition 1$)$ which implies $K_{e}(0)=(p+\theta)\left(t+\frac{1-p}{\theta} c\right)<c$, or $t \leq-\frac{1-p}{\theta} c\left(2(c)\right.$ in Proposition 1) which implies $K_{e}(0)=0<c$. 
Also, $K_{e}\left(\frac{p+\theta}{1-p-\theta}\right)=c+\frac{p}{\theta} c>c$. Since $K_{e}(\lambda)$ is increasing in $\lambda$, there is a cutoff $\hat{\lambda}<\frac{p+\theta}{1-p-\theta}$ for which there is too little incentive when $\lambda>\hat{\lambda}$ and too much incentive when $\lambda<\hat{\lambda}$.

Note that when $t<0$, the cutoff $\hat{\lambda}$ may be strictly more than 1 , which means there is a range of $\lambda$ over which punishment is more expensive than reward in moving the agent's payoff and yet the principal has too much incentive to motivate effort when punishment is available. Here is a numerical example. Suppose $p=0.4, \theta=0.4$, $t=-1, c=1, \lambda=1.2$ and $v=2.25$. This falls into the Subcase 2(b) in Proposition 1 . Therefore, the cost under the effort scheme is:

$$
(p+\theta)\left(t+\frac{1-p}{\theta} c\right)+(1-p-\theta)\left(\frac{p}{\theta} c-t\right) \lambda=0.8 \times 0.5+0.2 \times 2 \times 1.2=0.88 .
$$

Since $\theta v=0.9<1=c$, the efficient outcome is no effort. However, since $\theta v=0.9>0.88$, the principal chooses to offer the effort scheme.

\subsection{Altruistic Principal}

One form of the cost of punishment to the principal is simply the internalization of the agent's suffering. This is particularly relevant to the application of a parent-child relationship. The long term nature and the informality of the contractual relationship means that it is best studied with the framework in Section 3. The take-away from this discussion is that, as expected, more altruistic principal lowers the intensity of punishment and increases the intensity of reward. One reason is that reward becomes cheaper and punishment becomes more expensive to a more altruistic principal. The other reason is that the credibility upper bound for reward is relaxed, while that for the punishment does not change for a more altruistic principal.

I will parameterize the degree of the altruism by $\rho \in(0,1) \cdot{ }^{13}$ This is the percentage of the agent's payoff that is internalized by the principal. To fit the application of a parent-child relationship, I assume that the value of a good outcome $v$ accrues to the agent, but as a myopic child the agent does not internalize it, but the principal gets $\rho v$ from it. The principal also incur $\rho c$ because of the effort cost. The internalization means that any punishment $x_{1}<0$ suffered by the agent upon a bad outcome imposes a cost of $\rho\left(-x_{1}\right)$ on the principal. I assume there is no other cost of implementing the punishment. The internalization means the principal's cost of rewarding the agent is reduced by $\rho x_{2}$ from $x_{2}$.

Let's first evaluate the new credibility constraints:

$$
\begin{array}{r}
-\rho\left(-x_{1}\right)+\frac{\delta}{1-\delta}\left(\underline{v}+(p+\theta) \rho v-(p+\theta)(1-\rho) x_{2}-(1-p-\theta) \rho\left(-x_{1}\right)\right) \geq \frac{\delta}{1-\delta}(\underline{v}+p \rho v) \\
-(1-\rho) x_{2}+\frac{\delta}{1-\delta}\left(\underline{v}+(p+\theta) \rho v-(p+\theta) x_{2}-(1-p-\theta) \rho\left(-x_{1}\right)\right) \geq \frac{\delta}{1-\delta}(\underline{v}+p \rho v)
\end{array}
$$

This can be simplified into one credibility constraint:

$$
\max \left\{\frac{\rho}{1-\rho}(1+\alpha)\left(-x_{1}\right)+\beta x_{2}, \frac{\rho}{1-\rho} \alpha\left(-x_{1}\right)+(1+\beta) x_{2}\right\} \leq \frac{\rho}{1-\rho} \gamma
$$

where $\alpha, \beta$ and $\gamma$ are defined as in Section 3.

\section{The least costly way of implementing effort}

In this setting, the principal's problem of implementing the effort in the least costly way is:

$$
\begin{array}{ll}
\min _{x_{1}, x_{2}} & (1-p-\theta)\left(-x_{1}\right) \rho+(p+\theta) x_{2}(1-\rho)+\rho c \\
\text { s.t. } & \theta\left(x_{2}-x_{1}\right) \geq c \\
& \max \left\{\frac{\rho}{1-\rho}(1+\alpha)\left(-x_{1}\right)+\beta x_{2}, \frac{\rho}{1-\rho} \alpha\left(-x_{1}\right)+(1+\beta) x_{2}\right\} \leq \frac{\rho}{1-\rho} \gamma \\
& x_{1} \leq 0 \text { and } x_{2} \geq 0
\end{array}
$$

Note that this is equivalent to:

$$
\begin{array}{ll}
\min _{x_{1}, x_{2}} & (1-p-\theta) \lambda^{\prime}\left(-x_{1}\right)+(p+\theta) x_{2} \\
\text { s.t. } & \theta\left(x_{2}-x_{1}\right) \geq c \\
& \max \left\{\lambda^{\prime}(1+\alpha)\left(-x_{1}\right)+\beta x_{2}, \lambda^{\prime} \alpha\left(-x_{1}\right)+(1+\beta) x_{2}\right\} \leq \gamma^{\prime} \\
& x_{1} \leq 0 \text { and } x_{2} \geq 0
\end{array}
$$


where $\lambda^{\prime}=\frac{\rho}{1-\rho}$ and $\gamma^{\prime}=\frac{\rho}{1-\rho} \gamma$.

Therefore, the analysis of the optimal stationary scheme is the same as section 3 , except with $\lambda$ replaced by $\lambda^{\prime}$ and $\gamma$ replaced by $\gamma^{\prime}$.

I am interested in the comparative statics with respect to the degree of altruism $\rho$. Since $\lambda^{\prime}$ increases in $\rho$, as expected, a more altruistic principal faces higher cost of punishment and lower cost of reward, which calls for a higher intensity of reward and a lower intensity of punishment. However, a more altruistic principal also has a more relaxed credibility constraint because she cares more about the future value of effort, a more relaxed CC causes the punishment level to go up when CC is binding and when punishment is cheaper than reward. A more caring parent, for example, is simply more credible in carrying through a punishment than a less caring parent.

\subsection{Alternative Costs of Reward and Punishment}

In this section, I show the intuition extends to the case of the convex costs of punishments and rewards. Suppose, for example, the principal's cost is quadratic. That is, for $\lambda>0$ :

$$
k(x)=\left\{\begin{array}{l}
x^{2} \text { if } x>0 \\
\lambda(-x)^{2} \text { if } x \leq 0
\end{array}\right.
$$

It changes the objective function from Problem (*) but not the IC and IR constraints. When $t$ is sufficiently low so IR is not binding at the solution, the optimal scheme is:

$$
\begin{gathered}
x_{1}=-\frac{p+\theta}{(1-p-\theta) \lambda+(p+\theta)} \frac{c}{\theta} \\
x_{2}=\frac{(1-p-\theta) \lambda}{(1-p-\theta) \lambda+(p+\theta)} \frac{c}{\theta}
\end{gathered}
$$

Here, there is always mixing: the bad outcome will always get some punishment and the good outcome will always get some reward because a tiny amount of reward or punishment has an infinitely small marginal cost to the principal, which is not the case under linear costs. However, one can still see the same impact from $\theta$. A higher $\theta$ increases the first fraction in expression (1) while decreases the first fraction in expression (2): a better chance of success makes the reward more likely to be executed and thus more expensive in expectation and at the same time makes the punishment less likely to be executed thus cheaper in expectation. This shows the same incentive as in the linear model for the principal to replace rewards with punishments for an agent with higher benefit of effort. A higher $\theta$ at the same time makes the second fraction in both expressions (1) and (2) decrease. This is because a higher benefit of effort relaxes the IC constraint and calls for less powered incentives.

The agent's expected payoff from receiving the rewards alone is therefore:

$$
(p+\theta) x_{2}=(p+\theta) \frac{(1-p-\theta) \lambda}{(1-p-\theta) \lambda+(p+\theta)} \frac{c}{\theta}=\lambda\left(\frac{p}{\theta}+1\right) \frac{(1-p-\theta)}{(1-p-\theta) \lambda+(p+\theta)}
$$

Note that the term in the parentheses is decreasing in $\theta$ and the fraction is decreasing in $\theta$ as well. Therefore, the agent's expected payoff from rewards is decreasing in $\theta$. This reflects, as before, that the agent is rewarded less when the benefit of effort is higher. Meanwhile, the agent's expected payoff from receiving the punishments alone is:

$$
(1-p-\theta) x_{1}=-(1-p-\theta) \frac{p+\theta}{(1-p-\theta) \lambda+(p+\theta)} \frac{c}{\theta}=-\left(\frac{p}{\theta}+1\right) \frac{(1-p-\theta)}{(1-p-\theta) \lambda+(p+\theta)}
$$

This expected payoff is negative and the absolute value is decreasing in $\theta$. This reflects, as before, that the agent is punished less when the benefit of effort is higher.

The agent's expected payoff under the optimal scheme is the sum of the above two expressions, which are identical except for the scalar $\lambda$ and the sign in front. Therefore, we have:

\section{Proposition 4.}

Under quadratic principal's costs of rewards and punishments, the agent's expected payoff under the optimal scheme depends on $\lambda$ :

1. When $\lambda>1$, the agent's expected payoff under the optimal scheme is decreasing in $\theta$. 
2. When $\lambda<1$, the agent's expected payoff under the optimal scheme is increasing in $\theta$.

That is, when the punishments are more costly than rewards (per unit of agent's payoff), the principal prefers using rewards, so the comparative statics with respect to $\theta$ is dominated by the impact from rewarding the agent. On the other hand, when the punishments are less costly, the comparative statics with respect to $\theta$ is dominated by the impact from punishing the agent.

\subsection{Finitely Many Outcomes}

In this section I show that the model can generalize to $n>2$ outcomes. Under some assumptions, the only two relevant outcomes for the principal's optimal scheme are the worst and the best outcomes: the principal does not want to reward or punish any other outcomes.

There are $n$ outcomes, ranked from the worst to the best from the principal's perspective. When the agent exerts no effort, the probabilities of these outcomes are $\pi_{1}^{0}, \pi_{2}^{0}, \ldots, \pi_{n}^{0}$ respectively with $\Sigma_{i=1}^{n} \pi_{i}^{0}=1$. When the agent exerts the effort, the probabilities of these outcomes are $\pi_{1}, \pi_{2}, \ldots, \pi_{n}$ respectively with $\sum_{i=1}^{n} \pi_{i}=1$. Following Grossman and Hart (1983), I assume that all probabilities are strictly positive. I assume a strict version of the Monotone Likelihood Ratio Condition (MLRC) holds:

\section{Assumption 2.}

(MLRC) The ratio $\pi_{i} / \pi_{i}^{0}$ is increasing in $i$.

Let $q_{i} \equiv \pi_{i}-\pi_{i}^{0}$ denote the change in the probability of outcome $i$ induced by the effort. MLRC implies that $q_{1}<0, q_{n}>0$. I will refer to outcomes for which $q_{i}<0$ as negative outcomes, outcomes for which $q_{i}>0$ as positive outcomes and outcomes for which $q_{i}=0$ as neutral outcomes. That is, effort decreases the chance of negative outcomes and increases the chance of positive outcomes. The sets of all negative, neutral and positive outcomes are respectively consecutive outcomes. I first establish a useful property derived from MLRC:

\section{Lemma 2.}

For $i<j$, MLRC implies $\pi_{i} q_{j}>\pi_{j} q_{i}$.

\section{Proof.}

Consider $i<j$.

$$
\begin{array}{rc}
\frac{\pi_{i}}{\pi_{i}^{0}}< & \frac{\pi_{j}}{\pi_{j}^{0}} \\
\Leftrightarrow \pi_{i} \pi_{j}^{0}< & \pi_{j} \pi_{i}^{0} \\
\Leftrightarrow-\pi_{i} \pi_{j}^{0}> & -\pi_{j} \pi_{i}^{0} \\
\Leftrightarrow \pi_{i}\left(\pi_{j}-\pi_{j}^{0}\right)> & \pi_{j}\left(\pi_{i}-\pi_{i}^{0}\right) \\
\Leftrightarrow \pi_{i} q_{j}> & \pi_{j} q_{i}
\end{array}
$$

The principal's problem of implementing the effort in the least costly way is:

$$
\begin{array}{lll}
\min _{\left\{x_{i}\right\}_{i=1}^{n}} & \sum_{i=1}^{n} \pi_{i} k\left(x_{i}\right) & (* * *) \\
\text { s.t. } & \sum_{i=1}^{n} q_{i} x_{i} \geq c & \text { (IC) } \\
& \sum_{i=1}^{n} \pi_{i} x_{i} \geq c+t & \text { (IR) }
\end{array}
$$

If at the optimal scheme a negative outcome $i$ is given a costly reward $x_{i}>0$, then it must be that IR is binding. However, one can achieve the same objective function value by moving this reward to the best outcome $n$ by setting $x_{i}^{\prime}=0$ and $x_{n}^{\prime}=x_{n}+\pi_{i} x_{i} / \pi_{n}$. This will keep IR binding and strictly relaxes IC.

If at the optimal scheme a positive outcome $i<n$ is given a costly reward $x_{i}>0$. Consider a new scheme with $x_{i}^{\prime}=0$ and $x_{j}^{\prime}=x_{j}+\pi_{i} x_{i} / \pi_{j}$ for some $j>i$. That is, one removes the reward on outcome $i$ to a higher outcome $j$. This does not change the left-hand-side of IR. The left-hand-side of IC changes by $-q_{i} x_{i}+q_{j} \frac{\pi_{i}}{\pi_{j}} x_{i}=$ $\frac{x_{i}}{\pi_{j}}\left(\pi_{i} q_{j}-\pi_{j} q_{i}\right)>0$ by Lemma 2 . That is, IC is relaxed. Therefore, WLOG, I will restrict attention to schemes where reward is only given to the best outcome: outcome $n$. But keep in mind that every optimal scheme that rewards only the best outcome with no punishment on any other outcome can be changed into one where some other positive outcomes are also rewarded.

Because punishment is costly to both principal and agent, punishment is only used on negative outcomes. Moreover, punishment is only used when IC is binding. 


\section{Lemma 3.} $(* * *))$.

Only the worst outcome may receive a punishment in the optimal scheme to induce effort (the solution to Problem

\section{Proof.}

Suppose to the contrary, there exists a negative outcome $i>1$ that is given a punishment $x_{i}<0$. Consider an alternative scheme $x_{i}^{\prime}=x_{i}+\epsilon$ and $x_{1}^{\prime}=x_{1}-q_{i} \epsilon / q_{1}$ with $\varepsilon \in\left(0,-x_{i}\right)$. That is, one reduces the punishment on outcome $i$ and increases the punishment on outcome 1. This way, the left-hand-side of IC remains the same. The expected cost for the principal changes by.

$$
\lambda \pi_{1} q_{i} \epsilon / q_{1}-\lambda \pi_{i} \epsilon=\lambda \epsilon q_{1}\left(\pi_{1} q_{i}-\pi_{i} q_{1}\right)<0
$$

The inequality follows from Lemma 2 and the fact that $q_{1}<0$. That is, the expected cost of the principal is reduced. This also implies that the left hand side of IR increases by

$$
-\epsilon q_{1}\left(\pi_{1} q_{i}-\pi_{i} q_{1}\right)>0
$$

So IR is still satisfied. Therefore, this new scheme is better, which forms a contradiction.

Therefore, one can consider only schemes that punishes the worst outcome and rewards the best outcome. This finitely-many-outcome problem reduces to one with binary outcomes under the MLRC assumption and the linearity of the cost structure.

\section{Concluding Remarks}

In this paper, I recognize a principal's cost of using non-monetary punishments to motivate an agent to exert an effort. Punishments are often costly to the principal because of the resistance of the agent to the punishments or because the principal internalizes some of the agent's payoff. I investigate the optimal scheme for the principal in two frameworks. First, I derive insights on when to promise only rewards for a good outcome, when to promise only punishments for a bad outcome and when to promise both and how much of each to promise, taking into account the outside option of the agent. Second, I derive insights on how much reward or punishment or both to execute in each period when the principal repeatedly interacts with the agent where the agent can only be motivated by rewards and punishments that are credible. There are several major considerations for the principal. First, the optimal scheme depends on which of the reward or punishment is more cost effective. If effort is more likely to generate a good outcome, then punishment is more likely to be cost effective because of the lower chance of actually having to execute it. Second, the principal has to consider how to keep the agent happy enough to enter into a relationship with the principal, which sometimes calls for reward over punishment. Third, the principal has to consider whether her announcements of rewards and punishments are credible. Too high a reward or too high a punishment is not credible because of the ex post incentive to renege on them when the principal cannot fully commit. I show that, counter-intuitively, the agent's expected payoff under the optimal scheme may decrease when the benefit of the effort increases, because the principal optimally replaces rewards with punishments.

This paper has no private information. In the examples of the teacher-student, parent-child and firm-worker, the principal is also likely to be better informed of the ability of the agent or the nature of the task (which are related to $p$ and $\theta$ ). This will cause the reward/punishment scheme to have a signaling effect. It is intuitive that the agent will try to infer his ability or the task's difficulty from the scheme offered, which will affect the agent's motivation. The insights of this paper suggest that, depending on the parameter case, more punishment can signal a higher ability $(\theta)$ because a higher ability implies that the principal is more likely to avoid executing the costly punishment and it is cheaper to use punishment to motivate effort. ${ }^{14}$ Similarly, a higher intensity of reward can signal a lower ability, which is consistent with some findings in psychology. See Brummelman et al. (2014).

As mentioned in the introduction, the no-reward-no-punishment outcome can depend on the agent's expectation. This expectation is likely formed based on historical practices. The formation of this expectation and the resulting cost structure of the reward and punishment will be an interesting topic for future research: giving workers a low payoff this period may cause resentment and protest in the current period but will make it cheaper to reward the workers in the future. Giving children an hour of TV time today will make it a lot harder to give them only thirty minutes tomorrow.

As is often the case, the principal's cost of rewarding or punishing the agent is stochastic and not directly observable to the agent. For example, not picking up a crying baby that wakes up in the middle of the night is a 
common method to train the baby to sleep through the night. However, the parent's cost of that punishment is stochastic: it is higher, for instance, when an older child in the house is sick and needs more sleep or when the parents have significant work obligations during the day and thus need more sleep. Therefore, incorporating the costly non-monetary punishment in a repeated game with one-sided private information is immensely interesting for future research.

\section{Acknowledgements}

The author is grateful for comments from two anonymous referees, Dan Bernhardt, Jacques Cremer, Jin Li, Albert Ma, Wing Suen and the audience at International Industrial Organization Conference

\section{Appendix}

\section{A Proof of Lemma 1.}

\section{Proof.}

Suppose the optimal scheme has $x_{1}<0$ and $x_{2}<0$. Consider another scheme with $x_{1}^{\prime}=\left[(1-p-\theta) x_{1}+(p+\right.$ $\left.\theta) x_{2}\right] /(1-p-\theta)+\epsilon$ and $x_{2}^{\prime}=0$. Since IR is satisfied with $x_{1}, x_{2}$, IR must also be satisfied with $x_{1}^{\prime}$ and $x_{2}^{\prime}$ by construction for any $\varepsilon>0$. Since IC is satisfied with $x_{1}, x_{2}, x_{2}-x_{1}>\frac{c}{\theta}$. I have $x_{2}^{\prime}-x_{1}^{\prime}=x_{2}-x_{1}+\frac{1}{1-p-\theta}\left(-x_{2}\right)-\epsilon>$ $\frac{c}{\theta}+\frac{1}{1-p-\theta}\left(-x_{2}\right)-\epsilon$. Therefore, for any $\epsilon \in\left(0, \frac{1}{1-p-\theta}\left(-x_{2}\right)\right)$, the IC holds for $x_{1}^{\prime}, x_{2}^{\prime}$. Because the new scheme $x_{1}^{\prime}, x_{2}^{\prime}$ involves lower punishment for both outcomes than scheme $x_{1}, x_{2}$, the principal's cost is lower under $x_{1}^{\prime}, x_{2}^{\prime}$.

Suppose the optimal scheme has $x_{1}>0$ and $x_{2}>0$. Suppose IR is not binding. Consider another scheme with $x_{1}^{\prime}=0$ and $x_{2}^{\prime}=\left[(1-p-\theta) x_{1}+(p+\theta) x_{2}\right] /(p+\theta)-\epsilon>0$. By construction, it satisfies IR for $\varepsilon$ small enough. Since the original scheme satisfies IC, $x_{2}>x_{1}+\frac{c}{\theta}$. This implies $x_{2}^{\prime}-x_{1}^{\prime}>\frac{x_{1}}{p+\theta}+\frac{c}{\theta}-\epsilon>\frac{c}{\theta}$ if $\varepsilon$ is small enough, which satisfies IC. Then this new scheme $x_{1}^{\prime}, x_{2}^{\prime}$ reduces the cost to implement the effort, which is a contradiction. Therefore, IR has to binding.

Consider another scheme with $x_{1}^{\prime}=0$ and $x_{2}^{\prime}=\left[(1-p-\theta) x_{1}+(p+\theta) x_{2}\right] /(p+\theta)>0$. By construction, it also satisfies IR. Since the original scheme satisfies IC, $x_{2}>x_{1}+\frac{c}{\theta}$. This implies $x_{2}^{\prime}-x_{1}^{\prime}>\frac{x_{1}}{p+\theta}+\frac{c}{\theta}>\frac{c}{\theta}$, so the new scheme strictly relaxes IC. Therefore, $x_{1}^{\prime}, x_{2}^{\prime}$ implement the effort and results in the same cost to the principal.

Proof of Corollary 2, It follows from rewriting Proposition 1 in the following way:

\section{Lemma 4.}

Let $u_{A}$ denote the agent's expected payoff under the optimal scheme.

1. Under low outside option $t<-(1-p) c /\left(\frac{\lambda}{1+\lambda}-p\right) 0$,

a. if $\theta<\frac{\lambda}{1+\lambda}-p$, the scheme is pure reward and $u_{A}=\frac{p}{\theta} c>t$;

b. if $\theta>\frac{\lambda}{1+\lambda}-p$, the scheme is pure punishment and $u_{A}=-\frac{1-p}{\theta} c>t$.

2. Under intermediate outside option $-(1-p) c /\left(\frac{\lambda}{1+\lambda}-p\right)<t<0$,

a. if $\theta<\frac{\lambda}{1+\lambda}-p$, the scheme is pure reward and $u_{A}=\frac{p}{\theta} c>t$;

b. if $\frac{\lambda}{1+\lambda}-p<\theta<-\frac{1-p}{t} c$, the scheme uses both reward and punishment and $u_{A}=t$;

c. if $\theta>-\frac{1-p}{t} c$, the scheme is pure punishment and $u_{A}=-\frac{1-p}{\theta} c>t$.

3. Under intermediate outside option $0<t<p c /\left(\frac{\lambda}{1+\lambda}-p\right)$,

a. if $\theta<\frac{\lambda}{1+\lambda}-p$, the scheme is pure reward and $u_{A}=\frac{p}{\theta} c>t$;

b. if $\frac{\lambda}{1+\lambda}-p<\theta<\frac{p}{t} c$, the scheme uses both reward and punishment and $u_{A}=t$;

c. if $\theta>\frac{p}{t} c$, the scheme is pure reward and $u_{A}=t$.

4. Under high outside option $t>p c /\left(\frac{\lambda}{1+\lambda}-p\right)$, 
a. if $\theta<\frac{p}{t} c$, the scheme is pure reward and $u_{A}=\frac{p}{\theta} c>t$;

b. if $\theta>\frac{p}{t} c$, the scheme pure reward and $u_{A}=t$.

\section{Proof of Proposition 2.}

\section{Proof.}

As a preparation, we solve for the kink point of CC in the $\left(-x_{1}\right)-x_{2}$ space:

$$
\begin{aligned}
& \lambda(1+\alpha)\left(-x_{1}\right)+\beta x_{2}=\gamma \\
& \lambda \alpha\left(-x_{1}\right)+(1+\beta) x_{2}=\gamma
\end{aligned}
$$

This gives

$$
-x_{1}=\frac{1}{\lambda} \frac{\gamma}{1+\alpha+\beta}=\frac{\delta \theta v}{\lambda}, \quad x_{2}=\frac{\gamma}{1+\alpha+\beta}=\delta \theta v
$$

We consider two cases in turn.

Case 1. $\theta<\frac{\lambda}{1+\lambda}-p$. This case corresponds to Figure 1. The principal prefers rewards to punishments.

When $\frac{c}{\theta} \leq \frac{\gamma}{1+\beta}=\frac{\delta \theta v}{1-\delta(1-p-\theta)}$, the left most point on IC (hitting the vertical axis) satisfies CC. Therefore, the optimal scheme is pure reward: $x_{1}=0$ and $x_{2}=\frac{c}{\theta}$.

When $\frac{c}{\theta}>\frac{\gamma}{1+\beta}=\frac{\delta \theta v}{1-\delta(1-p-\theta)}$, IC intersects the upper part of CC as long as the kink point of CC is above or on IC. That is,

$$
\delta \theta v \geq \frac{c}{\theta}-\frac{1}{\lambda} \delta \theta v \Rightarrow \frac{c}{\theta} \leq \frac{1+\lambda}{\lambda} \frac{\gamma}{1+\alpha+\beta}=\frac{1+\lambda}{\lambda} \delta \theta v
$$

Otherwise, the solution does not exist and the effort cannot be motivated.

Solving for the intersection of IC and the upper part of CC:

$$
\begin{aligned}
\left(-x_{1}\right)+x_{2} & =\frac{c}{\theta} \\
\lambda \alpha\left(-x_{1}\right)+(1+\beta) x_{2} & =\gamma
\end{aligned}
$$

gives solution: $x_{1}=-\frac{(1+\beta) \frac{c}{\theta}-\gamma}{1+\beta-\lambda \alpha}=-\frac{(1-\delta(1-p-\theta)) \frac{c}{\theta}-\delta \theta v}{1-(1+\lambda) \delta(1-p-\theta)}$ and $x_{2}=\frac{\gamma-\lambda \alpha \frac{c}{\theta}}{1+\beta-\lambda \alpha}=\frac{\delta \theta v-\lambda \delta(1-p-\theta) \frac{c}{\theta}}{1-(1+\lambda) \delta(1-p-\theta)}$.

Case 2. $\theta>\frac{\lambda}{1+\lambda}-p$. The principal prefers punishments to rewards.

When $\frac{c}{\theta} \leq \frac{\gamma}{\lambda(1+\alpha)}=\frac{\delta \theta v}{\lambda(1-\delta(p+\theta))}$, the right most point of IC (hitting the horizontal axis) satisfies CC. Therefore, the optimal scheme is pure punishment: $x_{1}=-\frac{c}{\theta}$ and $x_{2}=0$.

When $\frac{c}{\theta}>\frac{\gamma}{\lambda(1+\alpha)}=\frac{\delta \theta v}{\lambda(1-\delta(p+\theta))}$, IC intersects the lower part of CC as long as the kink point of CC is above or on IC. As in Case 1, this condition is:

$$
\frac{c}{\theta} \leq \frac{1+\lambda}{\lambda} \delta \theta v
$$

Otherwise, the solution does not exist and the effort cannot be motivated.

Solving for the intersection of IC and the lower part of CC:

$$
\begin{aligned}
\left(-x_{1}\right)+x_{2} & =\frac{c}{\theta} \\
\lambda(1+\alpha)\left(-x_{1}\right)+\beta x_{2} & =\gamma
\end{aligned}
$$

gives solution: $x_{1}=-\frac{\gamma-\beta \frac{c}{\theta}}{\lambda(1+\alpha)-\beta}=-\frac{\delta \theta v-\delta(p+\theta) \frac{c}{\theta}}{\lambda-\delta(1+\lambda)(p+\theta)}$ and $x_{2}=\frac{\lambda(1+\alpha) \frac{c}{\theta}-\gamma}{\lambda(1+\alpha)-\beta}=\frac{\lambda(1-\delta)(p+\theta) \frac{c}{\theta}-\delta \theta v}{\lambda-\delta(1+\lambda)(p+\theta)}$.

\section{Notes}

1 The comparison of taxation and subsidy started from Bramhall and Mills (1966). See Polinsky (1979) for a review of this literature. There was a large empirical and experimental literature comparing the impacts of bonus and fines. See Nosenzo et al. (2013) for a review of this strand.

2 The U.S. Department of Justice estimated that local communities spent $\$ 22.2$ billion on jails in 2011. See Henrichson (2015).

3 Costly punishments will not be used if there is no limited liability: the principal can make the agent pay the principal which is a form of punishment that directly benefits the principal. 
4 This is different from some common use of the word "punishment". For example, Lazear (1991) argues that in the context of employment contracts, a bonus contract can easily be rewritten as a penalty contract by simply changing the baseline.

5 "Trump's Empty Threat to Stop Buying Saudi Oil", Reuters, November 6, 2016.

6 Sometimes the parents lay out the rules ahead of time as if offering a contract. Sometimes, the contract is implicit through repeated actions.

7 In Section 4.4 I show that the model can be easily generalized to finitely many outcomes under linearity of the principal's cost and a Monotone likelihood Ratio assumption.

8 It is sufficient to assume that the principal will choose to motivate effort even when costly punishment is not available $(\lambda \rightarrow \infty)$, which implies that she will motivate effort when $\lambda$ is finite. I discuss the case of lower $v$ and the possibility of the principal not motivating the effort in Section 4.1.

9 In Section 4.3, I discuss the impact of having nonlinear cost function for the principal.

10 The author's own childhood experience was that top students are harshly scolded when their test scores are less than perfect, while bottom students are praised for mediocre performances. Even though these practices are not formally written down, they are understood by the students.

11 So the outside option is no longer a force to generate a mixed scheme.

12 In contrast, Fong and $\mathrm{Li}$ (2017) show that when the agent and the principal are both somewhat patient, the optimal relational contract is not stationary. The principal is better off backloading the reward and the punishment after a "probation phase" during which the agent is not rewarded for a good outcome. The incentive to pay effort in these periods are sustained because the patient agent is motivated by the delayed future reward and punishment.

13 The case of full internalization $\rho=1$ is not interesting because it implies that reward is free to the principal and the principal is happy to pay the agent an infinite amount for a good outcome.

14 Writer Amy Chua wrote in her book "Battle Hymn of the Tiger Mother" (Chua 2011) about how her father called her "garbage" when she was not respectful to her mom. "My father angrily called me 'garbage'...I feel terrible and deeply ashamed of what I have done. But it didn't damage my self-esteem or anything like that. I knew exactly how highly he thought of me." The criticism from her parent allows her to update her belief of her own ability.

\section{References}

Baker, G., R. Cibbons, and K. J. Murphy. 1999. “Informal Authority in Organizations.” Journal of Law, Economics, and Organization 15 (1): 56-73. Burton, P., S. Phipps, and L. Curtis. 2002. "All in the Family: a Simultaneous Model of Parenting Style and Child Conduct." American Economic Review 92 (2): 368-72.

Bramhall, D. F., and E. S. Mills. 1966. “A Note on the Asymmetry Between Fees and Payments.” Water Resources Research 2 (3): 615-16.

Brummelman, E., S. Thomaes, B. O. de Castro, C. Overbeek, and B. J. Bushman. 2014. “That's Not Just Beautiful - That's Incredibly Beautiful!" The Adverse Impact of Inflated Praise on Children With Low Self-Esteem." Psychological science 25 (3): 728-35.

Chua, A. 2011. Battle Hymn of the Tiger Mother. London, England: Bloomsbury Publishing.

Dari-Mattiacci, G., and G. De Geest. 2010. “Carrots, Sticks, and the Multiplication Effect." Journal of Law, Economics, and Organization 26: 36567.

Fong, Y. F., and J. Li. 2017. “Relational Contracts, Limited Liability, and Employment Dynamics." Journal of Economic Theory 169: 270-93.

Gans, J. 2010. Parentonomics: An Economist Dad Looks at Parenting. Cambridge, MA, USA: MIT Press.

Henrichson, C., J. Rinaldi, and R. Delaney. 2015. The Price of Jails: Measuring the Taxpayer Cost of Local Incarceration. New York: VERA Institute of Justice. Retrieved online at: https://www.vera.org/publications/the-price-of-jails-measuring-the-taxpayer-cost-of-local-incarceration.

Kamijo, Y. 2016. "Rewards versus Punishments in Additive, Weakest-link, and Best-shot Contests." Journal of Economic Behavior and Organization 122: 17-30.

Larzelere, R. E., M. Klein, W. R. Schumm, and S.A. Alibrando Jr. 1989. “Relations of Spanking and Other Parenting Characteristics to Selfesteem and Perceived Fairness of Parental Discipline." Psychological Reports 64 (3c): 1140-42.

Lazear, E. P. 1991. “Labor Economics and the Psychology of Organizations.” Journal of Economic perspectives 5 (2): 89-110.

Lazear, E. P. 1995. Personnel Economics, vol. 1993. Cambridge, MA: MIT press.

Leshem, S., and A. Tabbach. 2015. "Solving the Volunteer's Dilemma: The Efficiency of Rewards Versus Punishments." American Law and Economics Review 18 (1): 1-32.

Levin, J. 2003. "Relational Incentive Contracts." American Economic Review 93 (3): 835-57.

$\mathrm{Li}$, J., and N. Matouschek. 2013. “Managing Conflicts in Relational Contracts.” American Economic Review 103 (6): 2328-51.

Li, J., N. Matouschek, and M. Powell. 2017. "Power Dynamics in Organizations." American Economic Journal: Microeconomics 9 (1): $217-41$.

Nosenzo, D., T. Offerman, M. Sefton, and A. van der Veen. 2013. "Encouraging compliance: Bonuses versus fines in inspection games." The Journal of Law, Economics, \& Organization 30 (3): 623-48.

Polinsky, A. M., 1979. "Notes on the Symmetry of Taxes and Subsidies in Pollution Control." The Canadian Journal of Economics/Revue canadienne d'Economique 12 (1): 75-83.

Wittman, D. 1984. “Liability for Harm or Restitution for Benefit?” The Journal of Legal Studies 13 (1): 57-80. 